\title{
ESTRESSE E RESILIÊNCIA NO TRABALHO EM SERVIDORES PÚBLICOS FEDERAIS*
}

Gabrieli Rossato ${ }^{1}$

Juliana Dal Ongaro ${ }^{1}$

Patricia Bitencourt Toscani Greco ${ }^{2}$

Emanuelli Mancio Ferreira da Luz

Luiza Dressler Sabin ${ }^{1}$

Tânia Solange Bosi de Souza Magnago ${ }^{1}$

\author{
https://orcid.org/0000-0002-4115-209X \\ https://orcid.org/0000-0003-0058-0132 \\ http://orcid.org/0000-0001-6999-5470 \\ https://orcid.org/0000-0002-7799-5232 \\ http://orcid.org/0000-0001-5665-9179 \\ http://orcid.org/0000-0002-5308-1604
}

Objetivo: Analisar a relação entre estresse e resiliência no trabalho em servidores públicos federais de uma instituição de ensino do Sul do Brasil. Método: Estudo transversal, realizado em 2018, com 526 servidores públicos federais de uma universidade do Rio Grande do Sul, Brasil. Utilizou-se questionário composto por variáveis sociodemográficas, laborais, hábitos e de saúde; e a Job Stress Scale e Resilience at Work Scale 20 - Brasil. Empregou-se análise estatística descritiva e multivariada. Para as associações, utilizaram-se os testes Qui-Quadrado de Pearson e Exato de Fisher, quando p<0,05, e a Regressão de Poisson. Foram seguidos os preceitos éticos da Resolução 466/2012. Resultados: Os servidores públicos apresentaram-se em trabalho ativo (23,5\%) e com médio nível de resiliência (38,8\%). Os servidores, em alta exigência (51\%) e em trabalho ativo (26\%), possuem prevalências mais elevadas para o baixo nível de resiliência no trabalho. Conclusão: Estar exposto a elevado estresse ocupacional pode influenciar negativamente no nível de resiliência do trabalhador.

Descritores: Estresse ocupacional; Resiliência; Trabalho; Saúde do trabalhador; Enfermagem.

\section{STRESS AND RESILIENCE AT WORK ON FEDERAL PUBLIC SERVERS}

Objective: To analyze the relation between stress and resilience at work in federal civil servants of an educational institution in the south of Brazil. Method: Cross-sectional study, conducted in 2018, with 526 federal civil servants from a university in Rio Grande do Sul, Brazil. A questionnaire composed of sociodemographic, work, habits and health variables was used; Job Stress Scale and Resilience at Work Scale 20 - Brazil. Descriptive and multivariate statistical analysis was used. For associations, Pearson's Chi-Square and Fisher's Exact tests were used when $\mathrm{p}<0.05$ and Poisson's Regression. The ethical principles of Resolution 466/2012 were followed. Results: Public servants presented themselves in active work (23,5\%) and with a medium level of resilience (38,8\%). Servants, in high demand (51\%) and in active work (26\%), have a higher prevalence for low level of resilience at work. Conclusion: Being exposed to high occupational stress can negatively influence the level of resilience of the worker. Descriptors: Occupational stress; Resilience; Job; Worker's health; Nursing.

\section{ESTRÉS Y RESILIENCIA EN EL TRABAJO SOBRE SERVIDORES PỦBLICOS FEDERALES}

Objetivo: Analizar la relación entre eles trés y la resiliencia en el trabajo en funcionarios federales de una institución educativa en el sur de Brasil. Método: Estudio transversal, realizado en 2018, con 526 funcionarios federales de una universidaden Rio Grande do Sul, Brasil. Se utilizó un cuestionario compuesto por variables sociodemográficas, laborales, de hábitos y de salud; Job Stress Scale y Resilience at Work Scale 20 - Brasil. Se utilizóun análisis estadístico descriptivo y multivariado. Para las asociaciones, se utilizaron las pruebas Chi-cuadrado de Pearson y Exacto de Fisher cuando $p<0.05$ y la regresión de Poisson. Se siguieron los principios éticos de la Resolución 466/2012. Resultados: Los servidores públicos se presentaron en un trabajo activo (23,5\%) y con un nivel medio de resiliencia (38,8\%). Los sirvientes, en alta demanda (51\%) y en trabajo activo (26\%), tienen una mayor prevalencia de bajo nivel de resiliencia en el trabajo. Conclusión: Estar expuesto a un alto estrés laboral puede influir negativamente en el nivel de resiliência del trabajador.

Descriptores: Estrés Laboral; Resiliencia Psicológica; Trabajo; Salud del trabajador; Enfermería.

*Artigo extraído de Trabalho de Conclusão de Curso apresentado à Universidade Federal de Santa Maria, em 2019.

'Universidade Federal de Santa Maria. Santa Maria-Rio Grande do Sul, Brasil.

2Universidade Regional Integrada do Alto Uruguai e das Missões. Santiago-Rio Grande do Sul, Brasil.

Autor Correspondente: Tânia Solange Bosi de Souza Magnago. E-mail: tmagnago@terra.com.br

Recebido: 14/4/20 Aceito: 16/5/20 


\section{INTRODUÇÃO}

O Ministério da Saúde, em 2012, instituiu a Política Nacional de Saúde do Trabalhador e da Trabalhadora com a finalidade de estabelecer princípios, diretrizes e estratégias com vistas à promoção e proteção à saúde do trabalhador ${ }^{1}$. A criação desta política denota a relevância do trabalho para a sociedade contemporânea, bem como a necessidade da manutenção do bem-estar no ambiente laboral. No entanto, as condições oferecidas pelo mercado de trabalho nem sempre têm desfechos favoráveis à saúde do trabalhador, o que pode propiciar um consumo demasiado da capacidade física e mental ${ }^{2-3}$.

Um dos principais fatores relacionados ao ambiente de trabalho que comprometem a saúde é o estresse ocupacional $^{4}$, definido como a relação entre altas demandas psicológicas e baixo controle sobre o trabalho ${ }^{5}$. No final dos anos de 1970, Karasek propôs o Modelo Demanda-Controle (Deman$d$-Control Model ou Job Strain), com vistas à investigação do estresse ocupacional. Ele se relaciona às demandas psicológicas e ao controle do trabalhador diante das atividades laborais, diferenciando quatro dimensões: alta exigência (alta demanda e baixo controle - pior situação); trabalho passivo (baixa demanda e baixo controle); baixa exigência (baixa demanda e alto controle - melhor situação) e trabalho ativo (alta demanda e alto controle $)^{5}$. Altas exigências laborais podem acarretar risco de adoecimento.

Os estressores advindos do ambiente laboral são fatores contribuintes para o surgimento de doenças como a síndrome metabólica, os distúrbios do sono, as enfermidades psicossomáticas, a síndrome de Burnout e a depressão ${ }^{6-7}$ Além disso, podem provocar a queda na produtividade, o absenteísmo, a insatisfação laboral e a baixa qualidade de vida ${ }^{6-7}$. Situações estressantes podem afetar profissionais de diferentes contextos laborais, como os da área da saúde ${ }^{8}$ e docentes ${ }^{9}$, tendo em vista suas características e desafios diários.

No contexto hospitalar, os profissionais da saúde representam algumas das categorias com maior vulnerabilidade para o estresse ocupacional ${ }^{6}$. Quer seja pela exposição diária a riscos químicos, físicos, ergonômicos, de acidentes por material biológico ou pelos riscos psicossociais como sobrecarga, precariedade das condições de trabalho e pressões exercidas pelos superiores, pacientes e seus familiares/acompanhantes $^{10}$. Estes fatores atingem os individuos e podem influenciar a atuação da equipe, gerando um quadro de insegurança para assistência à saúde ${ }^{11}$ que, por vezes, favorece o adoecimento dos trabalhadores ${ }^{12}$.

No contexto docente, também há evidências de profissionais sobrecarregados pelas demandas acadêmicas ${ }^{13}$, as quais podem ultrapassar sua carga horária. Somado ao desafio do ensino na graduação, tem-se o paralelo com a atuação nos programas de pós-graduação. As demandas se somam e as diversas exigências podem exacerbar o estresse ${ }^{13-14}$. Essa realidade pode implicar em exaustão física e emocional.

Em contraposição a esse cenário adverso, existem os aspectos positivos que permeiam o contexto laboral. Dentre estes, contempla-se a resiliência no trabalho, que está atrelada à capacidade do trabalhador de suportar e/ou superar as adversidades do meio e adaptar-se com sucesso à mudança e incerteza ${ }^{15}$. Esta capacidade é um aspecto que auxilia os trabalhadores no enfrentamento das situações estressoras, possibilitando efeitos benéficos sobre a saúde e o bem-estar destes. A resiliência no trabalho, quando investigada de forma adequada, possibilita desenvolver ações que auxiliem o trabalhador a enfrentar as adversidades da contemporaneidade e, consequentemente, melhorar as condições de saúde mental.

Frente ao exposto, tem-se como pergunta de pesquisa: Existe associação entre o estresse ocupacional e a resiliência no trabalho em servidores públicos federais? Neste intuito, delineou-se como objetivo, analisar a relação entre estresse e resiliência no trabalho em servidores públicos federais de uma instituição de ensino do Sul do Brasil.

\section{MÉTODO}

\section{Tipo de estudo}

Trata-se de um estudo epidemiológico transversal.

\section{Local de estudo}

O estudo foi desenvolvido em uma instituição pública de ensino do Rio Grande do Sul, Brasil, contemplando os seus oito centros de ensino superior, o colégio politécnico e técnico industrial, a unidade de educação infantil e o hospital universitário. Todos os centros e unidades dessa instituição foram incluidos para se ter representatividade nos resultados, fornecendo um panorama institucional sobre o estresse e a resiliência no trabalho.

\section{Participantes do estudo}

Este estudo está inserido em um projeto matricial intitulado "Adaptação transcultural e validação para a língua portuguesa do Brasil da Resilience at Work Scale (RAW Scale)". Foi desenvolvido com docentes (ensino superior, técnico e fundamental) e profissionais da saúde, todos servidores públicos da mesma universidade federal. Essa população foi selecionada a partir da similaridade com a utilizada no estudo de desenvolvimento da RAW Scale ${ }^{16}$, a fim de possibilitar a comparabilidade e a validação para a população. Além disso, é importante destacar que a população de docentes e profissionais da saúde estão cotidianamente expostos a riscos psicossociais no trabalho, o que traz à tona a relevância de investigações sobre resiliência nestas populações.

A população totalizou 2.866 docentes e profissionais da 
saúde. Realizou-se o cálculo amostral por meio de percentual estimado de $50 \%$, erro amostral de $5 \%$ e nivel de significância de $5 \%{ }^{17}$. A amostra foi aleatória e estratificada ${ }^{17}$ por centro/local, de forma que participaram do estudo 526 trabalhadores.

Foram estabelecidos como critérios de inclusão: ser docente de uma das unidades universitárias, técnica e tecnológica e da educação infantil da instituição, ou ser trabalhador da área da saúde do hospital universitário (médico, enfermeiro, técnico de enfermagem, fisioterapeuta, fonoaudiólogo, farmacêutico, assistente social, odontólogo, psicólogo ou nutricionista). Os critérios de exclusão foram: docentes e profissionais da saúde que estivessem em licença de saúde ou outro tipo de afastamento, no período de coleta dos dados, e os com tempo de trabalho na instituição inferior a um ano. Esta delimitação de tempo baseia-se no pressuposto de adaptação do trabalhador à instituição, especialmente no que tange à identificação dos estressores nas atividades laborais.

\section{Coleta de dados}

A coleta de dados foi realizada no período de abril a julho de 2018, por doutorandos, mestrandos e bolsistas de iniciação científica previamente capacitados pela coordenadora do projeto.

Inicialmente, a partir da lista de nomes dos participantes (professores e profissionais da saúde) foi realizado o sorteio para a composição de uma amostra estratificada por unidade universitária e, no hospital, por categoria profissional. Após, contatou-se via e-mail com os diretores dos centros e gestão hospitalar, para informar sobre a pesquisa e formalizar o convite.

O recrutamento dos participantes ocorreu individualmente e no próprio local de trabalho. Após o aceite em participar da pesquisa, os participantes foram informados sobre os objetivos, finalidade, riscos e benefícios do estudo e receberam o questionário auto preenchível composto por variáveis sociodemográficas, laborais, de hábitos e de saúde. Para avaliação do estresse ocupacional utilizou-se a Job Stress Scale $(\mathrm{JSS})^{18}$ e, para a avaliação da resiliência no trabalho, a RAW Scale 20 - Brasil ${ }^{19}$.

\section{Procedimentos de análise e tratamento dos dados}

Os dados foram inseridos no programa Epi-info ${ }^{\circledR}$, versão 6.4, com dupla digitação independente. Após a verificação de erros e inconsistências da digitação, os dados foram analisados no programa PASW Statistics ${ }^{\circledR}$ (Predictive Analytics Software, da SPSS Inc., Chicago, USA) 18.0.

As variáveis categóricas foram descritas por meio de frequência absoluta ( $N$ ) e relativa (\%). Já as quantitativas, pela média e desvio padrão (dados simétricos); pela mediana e intervalo interquartil (dados assimétricos), de acordo o teste Kolmogorov-Smirnov.
O estresse ocupacional percebido foi analisado pela JSS, composta por 11 questões, cinco para avaliar demanda psicológica do trabalho e seis para avaliar o controle sobre o trabatho. As opções de respostas variaram de "l" a "4", onde "Nunca ou quase sempre" pontuou 1 e "Frequentemente" pontuou 4. Os itens "4" e "9" da JSS foram reversos durante as análises. O escore das dimensões Demanda e Controle foram obtidos por meio da soma dos pontos atribuidos a cada uma das respostas que as contemplam.

O escore do domínio Demanda psicológica varia de "5" a "20" pontos ${ }^{18}$. Quanto maior o escore, maior a demanda. Ela foi dicotomizada pela mediana em "Baixa demanda" (5 a 14 pontos) e "Alta demanda" (15 a 20 pontos). Já o escore da dimensão Controle sobre o trabalho varia de 6 a 24 pontos $^{18}$. Quanto maior o escore, maior o controle. Foi dicotomizado pela mediana em "Baixo controle" (9 a 19 pontos) e "Alto controle" (20 a 24 pontos).

Os quadrantes do MDC foram obtidos pela combinação das categorias alta e baixa demanda psicológica e alto e baixo controle sobre o trabalho: alta exigência no trabalho (alta demanda psicológica e baixo controle - pior situação), trabatho ativo (alta demanda psicológica e alto controle), trabalho passivo (baixa demanda psicológica e baixo controle) e baixa exigência (baixa demanda psicológica e alto controle - melhor situação) $)^{18}$

A RAW Scale 20 - Brasil ${ }^{19}$ é composta por 20 itens, com opções de resposta em uma escala Likert de sete pontos, variando de "O" (Discordo totalmente) a "6" (Concordo totalmente) $)^{19}$. Os itens "9" e "11" foram reversos durante as análises. As análises seguiram o modelo da versão original ${ }^{16}$, que por direitos autorais não pode ser descrita. No entanto, seu uso para pesquisas acadêmicas pode ser solicitado pelo website: workingwithresilience.com.au, e/ou pelo endereço eletrônico contact@workingwithresilience.com.au.

A RAW Scale 20 - Brasil é um instrumento que possibilita investigar o grau de resiliência do trabalhador em sete fatores. A partir dos resultados, traçar estratégias em aspectos específicos do ambiente laboral, os quais poderão interferir de igual forma na resiliência global ${ }^{16,19}$.

Nas análises bivariadas, utilizaram-se os testes Qui-Quadrado de Pearson e Qui-Quadrado de Pearson com correção, quando indicado (células com menos de cinco indivíduos). $\mathrm{Na}$ multivariada, utilizou-se a Regressão de Poisson (bruta e ajustada) expressa na Razão de Prevalência e seus respectivos intervalos de confiança (IC95\%). Foram consideradas possiveis variáveis de confundimento aquelas que possuíam p-valor < 0,250 tanto para a exposição (estresse ocupacional), quanto para o desfecho (resiliência no trabalho). O nivel de significância adotado para todos os testes foi de $5 \%$. 
A análise da consistência interna da JSS e da RAW Scale 20 - Brasil foi analisada por meio do Coeficiente Alpha () de Cronbach. Valores $>0,70$ são considerados indicativos de consistência interna adequada ${ }^{20}$.

\section{Aspectos éticos}

Esta pesquisa foi aprovada pelo Comitê de Ética em Pesquisa da instituição pesquisada, sob Parecer no 2.121.699, e está de acordo com a Resolução no 466/2012, do Conselho Nacional de Saúde. Todos os que aceitaram participar assinaram o Termo de Consentimento Livre e Esclarecido (TCLE), em duas vias.

\section{RESULTADOS}

Dos 526 servidores públicos, 43,5\% eram docentes e $54,6 \%$, profissionais da saúde. Prevaleceram servidores do sexo feminino (64,6\%), com faixa etária entre 48 e 74 anos (36,3\%), casados/com companheiros (66,5\%) e de etnia branca $(92,2 \%)$.

Quanto à categoria profissional, predominaram trabalhadores docentes (45,4\%), seguidos dos técnicos de enfermagem $(16,9 \%)$ e de enfermeiros (11,8\%). A maior titulação foi a pós-graduação (especialização, mestrado e doutorado) (86,3\%), com carga horária semanal de 40 horas $(54,6 \%)$ e tempo de atuação na universidade e no hospital, em média, de 14,4 (DP=10,3) e 2,9 (DP=0,8) anos, respectivamente.

No que tange a hábitos e saúde, prevaleceram servidores que nunca fumaram (81,0\%), que às vezes utilizavam bebida alcoólica (66,3\%), e que, às vezes, possuíam tempo para lazer (48,7\%). Referiram não possuir alguma patologia diagnosticada (69,9\%), não fazer uso de medicação contínua (64,2\%), necessitar de atendimento médico no último ano $(51,6 \%)$ e não precisar de atendimento psicológico (77\%).

Quanto ao estresse ocupacional, prevaleceram servidores públicos com alta demanda psicológica (61,3\%) e alto controle sobre o trabalho (63\%). Na combinação dos quadrantes do MDC, 39,4\% estavam em Trabalho Ativo; 23,5\%, em baixa exigência; $22 \%$, em alta exigência; e 15,1\%, em trabalho passivo.

Conforme avaliação dos níveis de resiliência no trabalho, os participantes apresentaram moderado nível de resiliência no trabalho (38,8\%), seguido por alto nível (32,3\%) e baixo nível (28,8\%). A pontuação média foi de 71,94 (DP= 10,09 Mínimo= 38,33 e Máximo= 100)

A confiabilidade geral das escalas foi de 0,63 (JSS) e de 0,79 (RAW Scale 20 - Brasil).

Na Tabela 1, está apresentada a relação entre os níveis de resiliência no trabalho e o estresse ocupacional.

Tabela 1 - Distribuição da frequência de respostas da relação entre os níveis de resiliência no trabalho e o estresse ocupacional, RS, Brasil, 2018 ( $n=526)$.

\begin{tabular}{|c|c|c|c|c|c|c|}
\hline \multirow{3}{*}{$\begin{array}{l}\text { Estresse Ocupacional } \\
\text { Quadrantes do MDC* }\end{array}$} & \multicolumn{6}{|c|}{ Resiliência no trabalho } \\
\hline & \multicolumn{2}{|c|}{ Baixo } & \multicolumn{2}{|c|}{ Moderado } & \multicolumn{2}{|c|}{ Alto } \\
\hline & n & $\%$ & $\mathbf{n}$ & $\%$ & $\mathbf{n}$ & $\%$ \\
\hline Trabalho Passivo & 11 & 14,1 & 38 & 48,7 & 29 & 37,2 \\
\hline Alta Exigência** & 56 & 50,0 & 41 & 36,6 & 15 & 13,4 \\
\hline Baixa Exigência & 11 & 9,0 & 51 & 41,8 & 60 & 49,2 \\
\hline Trabalho Ativo & 72 & 35,1 & 71 & 34,6 & 62 & 30,2 \\
\hline
\end{tabular}

Nota: *MDC - Modelo Demanda-Controle. ** $\mathrm{p}<0,0001$ (Teste Qui-Quadrado). 
Trabalhadores em alta exigência apresentaram maior percentual para baixo nivel de resiliência no trabalho (50\%), comparados aos classificados em baixa exigência e alto nível de resiliência $(49,2 \% ; p<0,0001)$.
As associações brutas e ajustadas entre a resiliência no trabalho, estresse ocupacional e potenciais fatores de confundimento (faixa etária, tempo de formado e de trabalho, atendimento psicológico e tempo para o lazer) estão apresentadas na Tabela 2.

\begin{tabular}{|c|c|c|c|c|c|c|}
\hline Variáveis & $\mathbf{R P}_{\mathrm{b}}$ & IC (95\%) & $\mathbf{R P}_{\text {aj1 }^{*}}$ & IC (95\%) & $\mathbf{R P}_{\mathrm{aj}^{2 * *}}$ & IC (95\%) \\
\hline \multicolumn{7}{|l|}{ MDC } \\
\hline Alta Exigência & 1,70 & $1,41-2,05$ & 1,43 & $1,14-1,79$ & 1,51 & $1,24-1,85$ \\
\hline Trabalho Passivo & 1,23 & $0,96-1,57$ & 1,27 & $0,96-1,67$ & 1,15 & $0,90-1,48$ \\
\hline Trabalho Ativo & 1,37 & $1,12-1,67$ & 1,20 & $0,96-1,49$ & 1,26 & $1,03-1,55$ \\
\hline Baixa Exigência & 1,00 & -- & 1,00 & -- & 1,00 & -- \\
\hline \multicolumn{7}{|l|}{ Faixa etária (anos) } \\
\hline 38 a 47 & 1,21 & $1,04-1,41$ & 1,23 & $0,91-1,67$ & 1,08 & $0,94-1,25$ \\
\hline 23 a 37 & 1,20 & $1,03-1,40$ & 1,28 & $0,90-1,81$ & 1,14 & $0,98-1,31$ \\
\hline 48 a 74 & 1,00 & - & 1,00 & - & 1,00 & - \\
\hline \multicolumn{7}{|c|}{ Tempo de Formado (anos) } \\
\hline 12 a 22 & 1,21 & $1,04-1,41$ & 0,98 & $0,72-1,33$ & - & - \\
\hline 2 a 11 & 1,18 & $1,00-1,39$ & 1,07 & $0,75-1,53$ & - & - \\
\hline 23 a 48 & 1,00 & - & 1,00 & - & - & - \\
\hline \multicolumn{7}{|c|}{ Tempo de trabalho (anos) } \\
\hline 8 a 15 & 1,05 & $0,89-1,24$ & 0,91 & $0,73-1,13$ & - & - \\
\hline 1 a 7 & 1,08 & $0,91-1,28$ & 0,85 & $0,66-1,10$ & - & - \\
\hline 16 a 45 & 1,00 & - & 1,00 & - & - & - \\
\hline \multicolumn{7}{|c|}{ Atendimento Psicológico } \\
\hline Sim & 1,32 & $1,18-1,48$ & 1,17 & $1,03-1,33$ & 1,20 & $1,07-1,33$ \\
\hline Não & 1,00 & - & 1,00 & - & 1,00 & - \\
\hline \multicolumn{7}{|l|}{ Tempo para Lazer } \\
\hline Não & 1,36 & $1,10-1,68$ & 1,16 & $0,84-1,59$ & 1,21 & $0,97-1,51$ \\
\hline Às vezes & 1,34 & $1,18-1,53$ & 1,27 & $1,09-1,48$ & 1,24 & $1,08-1,41$ \\
\hline Sim & 1,00 & - & 1,00 & - & 1,00 & - \\
\hline
\end{tabular}

Legenda: $\mathrm{RP}_{\mathrm{b}}$ e $\mathrm{RP}_{\mathrm{aj}}=$ Regressão de Poisson bruta e ajustada.

Nota: ${ }^{*} P_{\text {ajl }}=$ resiliência + estresse ocupacional + faixa etária + tempo de formado + tempo de trabalho + atendimento psicológi$\mathrm{co}+$ tempo para o lazer. ${ }^{*} \mathrm{RP}_{\mathrm{aj} 2}=$ resiliência + estresse ocupacional + faixa etária + atendimento psicológico + tempo para o lazer.

Os servidores públicos em alta exigência e em trabalho ativo apresentaram $51 \%$ e $26 \%$, respectivamente, de prevalências mais elevadas para baixo nível de resiliência no trabalho, comparados aos em baixa exigência. Da mesma forma, ter necessitado de atendimento psicológico no último ano e às vezes ter tempo para o lazer apresentaram prevalências $20 \%$ e $24 \%$ mais elevadas para o baixo nível de resiliência no trabalho, respectivamente. 


\section{DISCUSSÃO}

Os trabalhadores estão sujeitos a presenciar o estresse em algum momento da vida. No entanto, algumas profissões apresentam maior exposição a condições desgastantes, tanto físicas quanto emocionais ${ }^{21-22}$. Nesse panorama, os profissionais da educação e da saúde possuem especificidades como sobrecarga, intensificação e condições deficitárias de trabalho.

Essas situações podem levar ao adoecimento, especialmente se o trabalhador possui baixa capacidade de adaptar-se e superar as situações adversas a que é exposto. No que tange a essa capacidade, os servidores públicos apresentaram moderado nível de resiliência no trabalho. Estudo que buscou conhecer os níveis de resiliência e Burnout, em 200 profissionais da enfermagem de hospitais públicos, evidenciou a maioria dos participantes (63\%) com resultados para moderada resiliência e que esta apresentou correlações negativas com a exaustão emocional e positivas com a realização pessoal ${ }^{23}$.

Estudos com docentes corroboram os achados aqui evidenciados ${ }^{24-25}$. Docentes de ensino superior de sete instituições públicas do México apresentaram pontuações médias ( $M d=3,22 ; \pm 0,70)$ para a variável mentalidade resiliente ${ }^{24}$. Outro, com docentes do ensino médio na Espanha, observou que eles possuiam média capacidade de identificar e gerenciar seus sentimentos e emoções, diante de certas condições e desafios ${ }^{25}$.

Nesse sentido, é possivel a reflexão de que situações adversas (sejam estas provenientes das condições de trabalho ou pessoais) podem interferir na capacidade do trabalhador de conseguir desenvolver e/ou permanecer resiliente em seu ambiente laboral. Desse modo, a resiliência pode ser intimamente influenciada pelas exigências e sobrecargas às quais os trabalhadores são expostos ${ }^{26}$.

Neste estudo, evidenciou-se que os trabalhadores que possuiam alta exigência apresentaram prevalência $51 \%$ mais elevada de não se tornarem resilientes. Diante disso, o modelo teórico de Karasek ${ }^{5}$ assevera que a alta exigência é caracterizada por elevadas demandas psicológicas e baixo controle sobre o trabalho. Nessa perspectiva, podem-se considerar os altos níveis de pressão (situação de emergência com pacientes para os profissionais da saúde e alta cobrança por produtividade para os docentes, por exemplo), de concentração (medo de errar, dimensionamento de pessoal inadequado; multitarefas; falta de acesso aos recursos necessários, por exemplo), estado de alerta e neces- sidade de aguardar as atividades dos outros (trabalho interdependente, por exemplo: esperar por resultados de exames ou de avaliação; entrega dos trabalhos por parte dos discentes), e não possuir espaços de aprendizagem (elevada carga horária; horas extras como fonte de renda, por exemplo), ou para exercerem sua criatividade e autonomia na tomada de decisões ${ }^{5}$

Essas características associadas à dificuldade de superar as situações laborais dificeis (baixa resiliência) favorecem a que o individuo desenvolva a maioria das reações adversas advindas das exigências psicológicas, como fadiga, ansiedade, depressão e doença física ${ }^{27-28}$. Isto pode implicar no mal-estar do trabalhador e na queda da sua produtividade destes.

Em relação ao trabalho ativo, evidenciou-se que o trabalhador que integrava esse quadrante apresentou significativamente uma prevalência $26 \%$ mais elevada para o baixo nível de resiliência no trabalho. Depreende-se disso que, mesmo o trabalhador possuindo alto controle sobre as suas atividades, estar constantemente ampliando sua capacidade para aprendizagem e motivação ${ }^{27}$ e estar submetido a elevadas demandas psicológicas, como o que acontece com os trabalhadores em alta exigência, é muito prejudicial. Tanto que ela reflete negativamente na capacidade de desenvolvera resiliência no meio laboral. Dessa forma, as chances de não desenvolver a capacidade resiliente são menores do que em alta exigência, embora ainda sejam significativas ${ }^{27}$.

Estudo realizado com funcionários efetivos, terceirizados e vigilantes de uma universidade pública constatou que a maioria dos trabalhadores apresentava nível baixo de estresse ocupacional nas três categorias (65\%, 49,2\% e 66,6\%, respectivamente) ${ }^{29}$. Desse modo, pode-se refletir que o desempenho das atividades laborais possui associação com suas competências e, quando possuem alto controle sobre o trabalho, acabam assumindo maiores demandas. Ainda, a exposição diária a diversos estressores pode desencadear reações nocivas ao individuo e, consequentemente, contribuir para o desenvolvimento de patologias ${ }^{29}$

Com relação à saúde mental dos trabalhadores da educação, em especial, professores, a Confederação Nacional dos Trabalhadores em Educação tem discutido sobre o tema. Essas discussões têm sido pautadas nos aspectos identificados como nocivos à saúde, a sobrecarga de trabalho e/ou a sua intensificação, juntamente com a falta de lazer, os quais estão fortemente associados com o estresse ocupacional desses 
trabalhadores ${ }^{30}$. Além disso, estudo evidenciou que as condições de trabalho relacionadas aos aspectos físicos, sociais e administrativos, também são fatores que impactam na saúde psíquica dos docentes ${ }^{31}$.

Dedicar um período para atividades de lazer é muito importante e contribui para o bem-estar físico e psiquico dos indivíduos. Estudos apontam que profissionais que desfrutam de atividades de lazer são expostos a benefícios em prol da qualidade de vida, como a redução do estresse, maior socialização e integração familiar, capacidade de reflexão e de resiliência ${ }^{32-33}$. No entanto, quando este trabalhador está exposto a situações estressoras em função das demandas excessivas de trabalho, e sem de exercer atividades de lazer, amplia-se o risco de adoecimento físico ou psíquico.

A Organização Mundial da Saúde (OMS) preconiza que a qualidade de vida pode ser entendida como o nível de percepção que o indivíduo tem diante da sua posição na vida, em relação à cultura, expectativas, preocupações e valores materiais ${ }^{34}$. Nesse sentido, estudo realizado com enfermeiros de um centro cirúrgico evidenciou que eles relacionavam diretamente a qualidade de vida com as condições de trabalho, com prática de atividade física, hábitos saudáveis e atividades de lazer $^{35}$. Dessa forma, a qualidade de vida, sendo indissociável do trabalho, sofre interferência deste, visto que ele pode potencializar a saúde ou o adoecimento ${ }^{35}$.

Nessa perspectiva, fortalecer não somente os aspectos físicos, mas também o psicológico dos trabalhadores pode ser considerado essencial para reforçar os aspectos que possibilitam a capacidade de resiliên$\mathrm{cia}^{36}$. Neste sentido, entende-se que manter a subjetividade do trabalhador, bem como seu tempo destinado para as atividades de lazer e prática de atividades de seu interesse, favorece positivamente a qualidade de $v^{2} a^{33}$ e o desenvolvimento da capacidade de resiliência no contexto do trabalho ${ }^{36}$.

\section{Limitações do estudo}

O delineamento transversal não possibilita inferir causalidade (viés da causalidade reversa), pois tanto desfecho quanto exposição foram avaliados no mesmo momento de tempo.

\section{Contribuições para a prática}

Os resultados deste estudo reforçam a importância das medidas preventivas e de promoção à saúde e resiliência do trabalhador, a fim de fortalecê-los física e emocionalmente. Também, de minimizar os agravos advindos deste meio, favorecendo um ambiente de trabalho saudável e positivo.

\section{CONCLUSÃO}

Os servidores em alta exigência e em trabalho ativo possuiam prevalências mais elevadas para o baixo nivel de resiliência no trabalho. Estas associações indicam que pode haver comprometimento da saúde do trabalhador, bem como proporcionam um panorama que possibilita o desenvolvimento de estratégias para reduzir as demandas psicológicas do trabalho e fortalecimento da resiliência.

Torna-se relevante destacar, que além dos resultados obtidos neste estudo, conhecer as particularidades do trabalhador e o contexto a que estão inseridos para exercer suas atividades, auxilia no direcionamento das estratégias a serem elaboradas. Assim, conhecer as associações entre estresse e resiliência, possibilitam o planejamento de ações de promoção da saúde e da resiliência de forma mais focada nas necessidades desta população, bem como a valorização, dos aspectos positivos do trabalhador.

\section{Contribuições dos Autores:}

Gabrieli Rossato participou na concepção e/ou desenho do estudo; coleta, análise e interpretação dos dados; redação e revisão crítica do manuscrito e aprovação da versão final a ser publicada; Juliana Dal Ongaro participou na concepção e/ou desenho do estudo; coleta, análise e interpretação dos dados; redação e revisão crítica do manuscrito e aprovação da versão final a ser publicada; Patrícia Bitencourt Toscani Greco participou na concepção e/ou desenho do estudo; redação e/ou revisão crítica do manuscrito e aprovação da versão final a ser publicada; Emanuelli Mancio Ferreira da Luz participou na concepção e/ou desenho do estudo; redação e/ou revisão crítica do manuscrito e aprovação da versão final a ser publicada; Luiza Dressler Sabin participou na concepção e/ou desenho do estudo; coleta, análise e interpretação dos dados; Tânia Solange Bosi de Souza Magnago participou na concepção e/ou desenho do estudo; coleta, análise e interpretação dos dados; redação e revisão crítica do manuscrito e aprovação da versão final a ser publicada.

\section{FINANCIAMENTO:}

Apoio financeiro: Edital № 009/2019 do Programa Institucional de Bolsas de Iniciação Científica (PIBIC-CNPq). 


\section{REFERÊNCIAS}

1. Ministério da Saúde (BR). Portaria no 1.823 de 23 de agosto de 2012. Institui a Política Nacional de Saúde do Trabalhador e da Trabalhadora. Diário Oficial da União. Brasilia, DF, 23 de agosto de 2012. [Internet]. 2012 [cited 2020 Fev 23]. Available from: https://bvsms.saude.gov.br/bvs/saudelegis/gm/2012/prt1823_23_08_2012.html.

2. Pialarissi R. Precarização do Trabalho. Adm Saúde. [Internet]. 2017 [cited 2020 Fev 23]; 17(66):1-12. Available from: http://cqh. org.br/ojs-2.4.8/index.php/ras/article/view/ll.

3. Araújo MRM, Morais KRS. Labor precariousness and the worker's overthrow process. Cad. Psicologia Social Trabalho [Internet]. 2017 [cited 2020 Fev23];20(1):1-13. Available from: http://www.revistas.usp.br/cpst/article/view/149091.

4. Lopes SL, Silva MC. Estresse ocupacional e fatores associados em servidores públicos de uma universidade federal do sul do Brasil. Ciênc. saúde coletiva [Internet]. 2018 [cited 2020 Fev23]; 23(11):3869-3880. Available from: http://www.scielo.br/scielo. php?script=sci_abstract\&pid=S1413-812320180011038698lng=pt\&nrm=iso.

5. Karasek RA. Job demands, job decision latitude, and mental strain: implications for job redesign. Adm. Sci. [Internet]. 1979; [cited 2020 Fev23]; 24(2):285-308. Available from: https://www.jstor.org/stable/2392498.

6. Ribeiro RP, Marziale MHP, Martins JT, Galdino MJQ, Ribeiro PHV. Occupational stress among health workers of a university hospital. Gaúcha Enferm. [Internet]. 2018 [cited 2020 Jan 20]; 39:e65127. Available from: http://www.scielo.br/scielo.php?pid=S1983-14472018000100421\&script=sci_arttext\&tlng=en.

7. Mininel VA, Felli VEA, Silva EJ, Torri Z, Abreu AP, Branco MTA. Psychic Workloads and Strain Processes in Nursing Workers of Brazilian University Hospitals. Latino-Am Enferm. [Internet]. 2013 [cited 2020 Jan 20];19(2):340-7. Available from: http://www. scielo.br/pdf/rlae/v2ln6/0104-1169-rlae-21-06-01290.pdf.

8. Negeliskii C, Lautert L. Occupational Stress and Work Capacity of Nurses of a Hospital Group. Rev Latino Am Enferm. [Internet]. 2011 [cited 2020 Jan 23]; 19(3):606-713. Available from: http://www.scielo.br/scielo.php?script=sci_arttextEpi$d=S 010411692011000300021$.

9. Araújo TM, Pinho PS, Masson MLV. Teachers' work and health in Brazil: thoughts on the history of research, strides, and challenges. Cad. Saúde Pública. [Internet]. 2019 [cited 2020 Fev 23]; 35(Sup 1):e00087318. Available from: https://www.scielo.br/pdf/ csp/v35sl/en_1678-4464-csp-35-sl-e00087318.pdf.

10. Ascari RA, Schmitz SS, Silva OM. Prevalência de doenças ocupacionais em profissionais da enfermagem: revisão de literatura. Rer Uningá. [Internet]. 2013 [cited 2020 Fev 23]; 15(2):26-31. Available from: http://revista.uninga.br/index.php/uningareviews/ article/view/1449.

11. Santana LC, Ferreira LA, Santana LPM. Occupational stress in nursing professionals of a university hospital. Rev Bras Enferm. [Internet]. 2020 [cited 2020 mar 15]; 73(2):e20180997. Available from: http://www.scielo.br/scielo.php?script=sci_arttextEpi$d=S 0034-71672020000200179$.

12. Gomez CM, Vasconcellos LCF, Machado JMH. A brief history of worker's health in Brazil's Unified Health System: progress and challenges. Ciênc. saúde coletiva. [Internet]. 2018 [cited 2020 mar 15]; 23(6):1963-1970. Available from: http://www.scielo.br/ scielo.php?pid=S1413812320180006019638script=sci_arttextEtlng=en.

13. Araújo BLS, Gomes DV, Pires VS, Moraes IM, Costa ALS. Estresse Ocupacional em Docentes de uma Instituição de Ensino Superior da região metropolitana de Goiânia. Revisa. [Internet]. 2015 [cited 2020 mar 15]; 4(2):96-104. Available from: http:// revistafacesa.senaaires.com.br/index.php/revisa/article/view/243.

14. Oliveira MGM, Cardoso CL. Stress e trabalho docente na área da saúde. Estud psicol. [Internet]. 2011 [cited 2020 mar 15]; 28(2):135-41. Available from: http://www.scielo.br/scielo.php?script=sci_arttext\&pid=S0103-166X2011000200001.

15. McEwen K. Building Resilience at work. 1르 Ed. Australian: Australian academic press. 2011.

16. Winwood PC, Colon R, Mcewen K. A pratical a measure of workplace resilience: Developing the Resilience at work scale. Journal of occupational and environmental medicine. American College of Occupational and Environmental Medicine. [Internet]. 2013 [cited 2020 mar 15]; 55(10):1205-1212. Available from: https://journals.lww.com/joem/Abstract/2013/10000/A_Practical_Measure_of_Workplace_Resilience_.12.aspx?trendmd-shared=0.

17. Barbetta PA. Estatistica aplicada às Ciências Sociais. 9 ạ Ed, Florianópolis: Editora da UFSC; 2015.

18. Alves MGM, Chor D, Faerstein E, Lopes CS, Werneck GL. Short version of the "job stress scale": a Portuguese-language adaptation. Rev Saúde Pública. [Internet]. 2004 [cited 2020 mar 15]; 38(2):1-7. Available from: http://www.scielo.br/pdf/rsp/v38n2/ en_19774.pdf 
19. Greco PBT. Adaptação transcultural e validação para a lingua portuguesa do Brasil da Resilience at Work Scale (RAW SCALE). [Tese] Universidade Federal de Santa Maria (UFSM, RS), 2018. [Internet]. 2018 [cited 2020 Fev 23]. Available from: https://repositorio.ufsm. br/bitstream/handle/1/16353/TES_PPGENFERMAGEM_2018_GRECO_PATRICIA.pdf?sequence=1EisAllowed=y.

20. Field A. Descobrindo a estatística usando o SPSS. 2a Ed. Porto Alegre: Artmed; 2009.

21. Magnago TSBS, Lisboa MTL, Griep RH, Zeitoune GRC, Tavares JP. Working conditions of nurses: evaluation based on the demand-control model. Acta Paul Enfermagem. [Internet]. 2010 [cited 2020 mar 10]; 23(6):811-7. Available from: http://www.scielo.br/scielo. php?pid=S010321002010000600015\&script=sci_arttextEtlng=en.

22. Weber LND, Leite CR, Stasiak GR, Santos CA da S, Forteski R. O estresse no trabalho do professor. Imag Educação. [Internet]. 2015 [cited 2020 mar 10]; 5(3):40-52. Available from: http://periodicos.uem.br/ojs/index.php/ImagensEduc/article/view/25789.

23. Silva SM, Borges E, Abreu M, Queirós C, Baptista P. Fell V. Relação entre resiliência e burnout: Promoção da saúde mental e ocupacional dos enfermeiros. Rer Port Enferm de Saúde Mental. [Internet]. 2016 [cited 2020 mar 10]; 2(16):41-48. Availablefrom: http:// www.scielo.mec.pt/scielo.php?script=sci_arttextEpid=\$1647-21602016000300006.

24. Hernández LMS, Macias AB, Bañalesc DLG. Mentalidad Resiliente y Salud Mental Positiva en docentes de Nivel Superior. INNODOCT. [Internet]. 2018 [cited 2020 mar 20]; 6:501-510. Available from: http://ocs.editorial.upv.es/index.php/INNODOCT/INN2018/paper/ viewFile/8802/4446

25. Vera IV, Gambarte MIG. Burnout and Resilience Factors in High School Teachers. International Journal of Sociology of Education. [Internet]. 2019 [cited 2020 mar 20]; 8(2):127-152. Available from: https://hipatiapress.com/hpjournals/index.php/rise/article/ view/3987.

26. Souza RG, Lindemam AC, Tsuge DM, Fontoura GA, Pargon JM, Santos KA. Saúde psiquica, resiliência e bem-estar do trabalhador: intervenção com profissionais da educação. Rev Amazônica. [Internet]. 2019 [cited 2020 mar 15]; 23(1):394-408. Available from: https://periodicos.ufam.edu.br/index.php/amazonica/article/view/5659/4357.

27. Urbanetto JS, Magalhães MCM, Maciel VO, Sant'Anna VM, Gustavo AS, Figueiredo CEP, Magnago TSBS. Work-related stress according to the demand-control model and minor psychic disorders in nursing workers. Rev Esc Enfermagem. [Internet]. 2013 [cited 2020 mar 15]; 47(3): 1180-1186. Available from: http://www.scielo.br/pdf/reeusp/v47n5/0080-6234-reeusp-47-05-1180.pdf.

28. Alves MGM, Braga VM, Faerstein E, Lopes CS, Junger W. The demand-control model for job strain: a commentary on different ways to operationalize the exposure variable. Cad. Saúde Pública. [Internet]. 2015 [cited 2020 mar 15]; 31(1):1-5. Available from: http://www. scielo.br/scielo.php?script=sci_arttextEpid=S0102311X2015000100208\&lng=en\&nrm=iso\&tlng=pt.

29. Cavalcante JL, Pinto AGA, Junior FEB, Moreira MRC, Lopes MSV, Cavalcante EGR. Estresse ocupacional dos funcionários de uma Universidade pública. Enferm. Foco. [Internet]. 2019 [cited 2020 mar 15]; 10(4):108-115. Available from:http://revista.cofen.gov.br/ index.php/enfermagem/article/view/2310.

30. Gouvêa LAVN. Teachers' working conditions and illnesses on the agenda of a labor Union. Rev Saúde Debate. [Internet]. 2016 [cited 2020 mar 15]; 40(1ll):206-219. Available from: http://www.scielo.br/pdf/sdeb/v40nlll/en_0103-1104-sdeb-40-1ll-0206.pdf.

31. Pinto MJS, Pintor FA, Detta FP. Condições de trabalho que mais impactam na saúde dos docentes de enfermagem: revisão integrativa. Enferm Foco. [Internet]. 2017 [cited 2020 mar 15]; 8(3):51-55. Available from: http://revista.cofen.gov.br/index.php/enfermagem/article/view/1239/399.

32. Costa MAR, Souza VS, Dias J, Cussunoque L, Francine G, Francisqueti V. Concepção dos profissionais de serviço de emergência sobre qualidade de vida. Ciên Biológicas e da Saúde. [Internet]. 2017 [cited 2020 mar 20]; 38(1):35-44. Available from: http://www.uel. br/revistas/uel/index.php/seminabio/article/view/25537.

33. Trapé AA, Lizzi EAS, Jacomini AM, Bueno CRJ, Franco LJ, Zago AS. Exercício físico supervisionado, aptidão física e fatores de risco para doenças cardiovasculares em adultos e idosos. Rev. Bras Ciências Da Saúde. [Internet]. 2018 [cited 2020 mar 20]; 22 (4):291-298. Available from: https://periodicos.ufpb.br/index.php/rbcs/article/view/34964-l.

34. Freire MN, Costa ER. Qualidade de vida dos profissionais de enfermagem no ambiente de trabalho. Rev. Enferm Contemporânea. [internet]. 2016 [cited 2020 mar 20]; 5(1):1-8. Available from: https://www5.bahiana.edu.br/index.php/enfermagem/article/view/871.

35. Santos DAC, Morais DSVD, Franco RVB, Gomes JRAA. Qualidade de vida sob a ótica de enfermeiros do centro cirúrgico de um Hospital público. Enferm Foco. [Internet]. 2019 [cited 2020 mar 20]; 10(4):07-11. Available from: http://revista.cofen.gov.br/index.php/ enfermagem/article/view/1676.

36. Amaral JF, Ribeiro JP, Paixão DX. Qualidade de vida no trabalho dos profissionais de enfermagem em ambiente hospitalar: uma revisão integrativa. Rev Espaço para a Saúde. [Internet]. 2015 [cited 2020 mar 20]; 16(1):66-74. Available from: https://www.researchgate.net/publication/316177620_Qualidade_de_vida_no_trabalho_dos_profissionais_de_enfermagem_em_ambiente_hospitalar_uma_revisao_integrativa. 